\title{
Acute Pain Predictors of Remote Postoperative Pain Resolution After Hand Surgery
}

Jennifer M. Hah (D) C Chinwe A. Nwaneshiudu • Eric M. Cramer •

Ian R. Carroll · Catherine M. Curtin

Received: March 2, 2021 / Accepted: March 31, 2021 / Published online: April 18, 2021

(C) The Author(s) 2021

\section{ABSTRACT}

Introduction: Chronic postsurgical pain (CPSP) is a global issue with high prevalence. This study compared acute pain descriptors among patients undergoing carpal tunnel release (CTR) or trigger finger release (TFR). We hypothesized worst pain intensity on postoperative day (POD) 10 would be best to predict the time to pain resolution.

Methods: In this secondary analysis of a negative, randomized, double-blind placebo-controlled trial, adult veterans undergoing CTR or TFR were enrolled January 2012-January 2014, with data analysis February 2020-October 2020. Participants were randomized to receive minocycline $200 \mathrm{mg}$ or placebo $2 \mathrm{~h}$ prior to the operation, then minocycline $100 \mathrm{mg}$ or placebo twice daily for 5 days. The Brief Pain Inventory,

J. M. Hah $(\bowtie)$. C. A. Nwaneshiudu · I. R. Carroll Division of Pain Medicine, Department of Anesthesiology, Perioperative, and Pain Medicine, Stanford University, 1070 Arastradero Rd, Ste 200, Palo Alto, CA 94304, USA

e-mail: jhah@stanford.edu

\section{E. M. Cramer}

Systems Neuroscience and Pain Lab, Stanford

University, Stanford, CA, USA

C. M. Curtin

Division of Hand and Plastic Surgery, Department of Orthopaedic Surgery, Stanford University, Stanford, CA, USA assessed daily, captured three pain scores: average and worst pain over the past $24 \mathrm{~h}$, and current pain intensity. Fifteen acute pain descriptors based on the pain scores (clusters, mean, median, pain scores on POD 10, and linear slopes) were compared as predictors of time to pain resolution.

Results: Of 131 randomized participants, 114 (83 CTR, 31 TFR) were included. Average pain over the last $24 \mathrm{~h}$ reported on POD 10 best predicted time to pain cessation. Every onepoint increase in the average pain score was associated with a $36.0 \%$ reduced rate of pain cessation (HR, 0.64, 95\% CI 0.55-0.74, $p<0.001)$. Average pain on POD 10 was significantly associated with the development of CPSP at 90 days (OR 1.74, 95\% CI 1.30-2.33, $p$ value $<0.001)$. The optimal cutoff score for the high-risk group was determined as average pain on POD $10 \geq 3$.

Conclusions: This study validates prior work and demonstrates the importance of assessing pain severity on POD 10 to identify patients at high risk for CPSP who are most likely to benefit from early pain intervention. Future research in diverse surgical cohorts is needed to further validate pain assessment on POD 10 as a significant predictor of CPSP.

Keywords: Acute pain; Carpal tunnel release; Chronic postsurgical pain; Hand surgery; Minocycline; Pain trajectories; Persistent 
postoperative pain; Postoperative pain; Trigger finger release

\section{Key Summary Points}

\section{Why carry out this study?}

The prevalence of severe chronic postsurgical pain (CPSP) is $10 \%$ across a range of operations, and $22 \%$ of patients undergoing hand surgery develop CPSP 1 year after surgery.

Identifying an immediate postoperative predictor of remote pain resolution occurring months after surgery has important clinical implications for the management of acute pain and prevention of CPSP.

We hypothesized that, by comparing 15 acute pain descriptors among patients undergoing CTR or TFR, worst pain reported on postoperative day (POD) 10 would best predict time to pain resolution.

What was learned from the study?

Assessment of pain intensity on POD 10 best predicts remote time to pain resolution in patients undergoing minor hand surgery under local anesthesia. Early identification of patients at high risk for CPSP allows for early intervention, closer follow-up, and initiation of multimodal pain therapy.

Average pain intensity reported on POD 10 best predicted time to pain resolution and was significantly associated with the development of CPSP 90 days after surgery. Patients at high risk for the development of CPSP reported average pain on $P O D 10 \geq 3$.

\section{DIGITAL FEATURES}

This article is published with digital features, including a summary slide, to facilitate understanding of the article. To view digital features for this article go to https://doi.org/10.6084/ m9.figshare.14340404.

\section{INTRODUCTION}

The prevalence of chronic postsurgical pain (CPSP) severe enough to cause functional limitations is $10 \%$ across a wide variety of operations [1]. CPSP is more common than most other postoperative complications and often results in long-term consequences. Thus, the global impact of CPSP on healthcare resource utilization remains enormous.

CPSP has a particularly high prevalence after major operations such as mastectomy, thoracotomy, or total knee arthroplasty, but even minor operations can result in CPSP. For example, extremely common hand procedures such as carpal tunnel release (CTR) and trigger finger release (TFR) result in a 20\% rate of CPSP 1 year after surgery [2]. Although patients undergoing CTR often do not receive general anesthesia, and the operation is minor, the incidence of CPSP is comparable to that of major operations [3, 4]. The burden of CPSP is also reflected in duration of postoperative opioid use after hand surgery. Among patients not taking preoperative opioids who underwent common hand surgery procedures including CTR and TFR, $13 \%$ of patients continued to fill opioid prescriptions 90 days after their operation [5]. Thus, CPSP is a universal surgical complication and reducing the incidence of CPSP will play a role in optimizing opioid use after surgery.

Early postsurgical pain is an important factor in the course of CPSP. The severity of acute pain after major operations has been associated with the development of CPSP [6]. The presence of pain in the first 3 days after CTR was significantly associated with the CPSP persisting 1 year after CTR [2]. However, only a minority of patients who experience severe acute pain will progress to experience CPSP, and other factors than the severity of acute and subacute pain may better predict which patients go on to develop chronic pain after surgery. Characterization of postoperative pain trajectories through postoperative days 5-6 may yield more 
granularity in describing a patient's acute pain experience to include both pain resolution and pain severity [7]. Other work shows that surgical patients may report a non-linear trajectory [2] with increasing pain in the subacute postoperative phase perhaps signaling the development of neuropathic pain and/or initiation of central sensitization [8]. Thus, analysis of acute postoperative pain trajectories limited to the first postoperative week may miss important information on who is likely to develop CPSP. Hand surgery is increasingly performed in the ambulatory setting [9], so remote assessments to aid in early identification of high-risk patients are needed. Once patients at high risk for prolonged pain, prolonged opioid use, and delayed recovery are identified, early intervention and targeted resource allocation can be implemented.

Of 15 acute postsurgical pain descriptors evaluated with data-mining algorithms, worst pain intensity over the previous $24 \mathrm{~h}$ assessed on POD 10 was significantly associated with time to pain resolution, opioid cessation and complete surgical recovery [10]. Metrics such as this may allow identification of patients at high risk for prolonged pain, prolonged opioid use, and delayed recovery in the subacute phase after surgery to be targeted for early intervention and more intensive resource allocation. The subacute postoperative phase lasting several weeks after surgery has been minimally characterized and recovery during this period warrants further research [8].

The aim of this study was to replicate this prior work in a cohort having minor surgery under local anesthesia. This study conducted a secondary analysis of a double-blind randomized controlled trial of 131 patients undergoing CTR or TFR under local anesthesia. This clinical trial assessed the effect of perioperative minocycline vs. placebo on time to pain resolution. Minocycline did not reduce time to pain resolution and appeared to increase time to pain resolution among patients with elevated posttraumatic stress disorder symptoms [11]. We used previously reported acute pain cluster centroids based on the first ten daily pain scores after surgery [10] to categorize patients into high vs. low acute pain clusters. We compared these clusters with 12 additional acute pain descriptors of postoperative pain intensity over the first 10 postoperative days to determine which descriptor best estimated the probability of time to pain resolution. Our aim was to characterize acute pain after hand surgery and replicate prior research findings of the optimal acute pain descriptor predicting the likelihood and speed of pain resolution, this time in a novel cohort of patients undergoing CTR or TFR under local anesthesia. We hypothesized that worst pain intensity over the prior $24 \mathrm{~h}$ assessed on POD 10 would be the best predictor of time to pain resolution among patients undergoing minor hand surgery.

\section{METHODS}

We conducted a secondary analysis of a randomized, double-blind placebo-controlled trial of adult veterans scheduled for CTR or TFR at a single center (VA Palo Alto Health Care System Hospital). Participants were randomized to receive either minocycline $200 \mathrm{mg}$ or placebo 2 $\mathrm{h}$ prior to the operation. They received either minocycline $100 \mathrm{mg}$ twice daily or placebo for 5 days at the same intervals. The study was approved by the Stanford University IRB \#21,794 (clinicaltrials.gov identifier NCT02051296, RX000487) and all patients provided informed consent to participate. A detailed description of the study protocol, demographics, measures, and methods has been published [11]. The study was performed in accordance with the Helsinki Declaration of 1964, and its later amendments. Participants were enrolled between January 2012 and January 2014. Data analysis for the present study was performed from February 2020 to October 2020 .

\section{Assessments}

Participants completed preoperative assessments in-person, and postoperative assessments via phone calls. The Brief Pain Inventory (BPI) is a validated measure of pain and pain interference of physical functioning measured on 0-10 numeric scales [12]. The Screen for 
Posttraumatic Stress Symptoms (SPTSS) consists of 17 items, and measures PTSD symptoms. SPTSS is intended to be a brief, validated, selfreport screening tool for psychological distress associated with trauma [13]. SPTSS scores were dichotomized to greater than or equal to 16 , or below 16 to denote the presence or absence of PTSD, respectively [11]. The Beck Depression Inventory-II (BDI-II) is a validated measure of depressive symptoms, and scores range from 0 to 63 with higher scores representing higher depressive symptoms [14]. The QuickDASH measures patient-reported upper-extremity physical function and disability, consists of 11 items, with scores ranging from 0 (best function) to 100 (worst function) [15]. The Short Form Health Survey (SF-36) consists of qualifyof-life measures covering eight domains of health. Physical and mental component summary scores of the SF-36 were calculated [16]. Preoperative assessments included the SF-36, QuickDASH, BPI, SPTSS, and BDI.

After surgery, participants were called starting POD 1 daily up to 3 months, and then weekly thereafter up to 2 years after the operation until pain resolution. A modified BPI was assessed at each call to assess pain at the surgical site, analgesic use, and recovery. This amounted to 3917 postoperative phone assessments.

\section{Operation}

All patients underwent either open CTR or open TFR with local anesthesia only. Both operations consisted of longitudinal incisions extending through the palmar fascia, closed with nylon sutures. Sutures were removed at POD 12.

\section{Study Outcomes}

The primary outcome was time to pain resolution defined as the number of days between surgery and the first of three consecutive days of 0 of 10 average pain at the surgical site assessed with the BPI [12].

\section{Statistical Analysis}

\section{Postoperative Acute Pain Cluster Analysis}

Previously reported cluster centroids [1], from a separate non-Veteran mixed surgical cohort, were used to assign patients to high and low acute pain clusters based on postoperative pain scores assessed over the first 10 days post-surgery. During each call, the patient reported their average and worst pain intensity over the preceding $24 \mathrm{~h}$ and current pain intensity at the surgical site. Each category of pain scores (average, current, worst) was examined as an acute pain trajectory, and each patient had three distinct trajectories representing the average, current, and worst pain scores over the first ten postoperative days. Cluster assignment was determined through Euclidean distance (the square root of the sum of each dimensional distance squared) between values and cluster centroids at each time point [10].

\section{Cox Proportional Hazard Regression and Sensitivity Analyses}

Time to pain resolution was analyzed using Cox proportional hazards regression. Given the possibility of different underlying hazard rates attributed to CTR vs. TFR, all subsequent analyses controlled for the type of operation. Fifteen acute pain descriptors (including average, current, and worst acute pain clusters; mean and median of each set of pain scores over the first ten postoperative days; and average, current, and worst pain scores reported POD 10) were evaluated as predictors of time to pain resolution. The linear slope of each acute pain trajectory was calculated for each patient and also evaluated as one of the acute pain descriptors. Model efficiency was determined with lower Akaike information criteria scores indicating the model with the best fit. Subgroup analyses for the optimal acute pain descriptor were conducted for treatment group, operation type, and presence of PTSD. Additional sensitivity analyses included examining the interaction of treatment group (minocycline vs. placebo) and presence of PTSD symptoms per the previously reported clinical trial findings in the best-fit 
model [11]. The preceding data analysis was conducted using R 4.0.2 (R Foundation).

\section{Determining Optimal Cutoff Value for the Best Acute Pain Descriptor}

SAS software, version 9.4 (SAS Institute Inc) was used for this analysis. A receiver operating characteristic (ROC) curve analysis was conducted examining the association of the best acute pain descriptor with persistent post-surgical pain continuing 90 days after surgery using the PROC LOGISTIC function in SAS. The AUC was determined for the best acute pain descriptor. Based on examination of the maximum Youden index $(J)$ value, minimum $D$ value (minimum distance to the ideal point of the ROC plot), and the equality point (minimum difference between the sensitivity and specificity), an optimal cut-off for the best acute pain descriptor was determined.

\section{Modified Poisson Regression Analysis of the Highest-Risk Patient Cluster}

Based on the optimal cut-off for the best acute pain descriptor, a high- vs. low-risk group for CPSP was identified. Continuous variables were compared with two-tailed, unpaired $t$ tests, and categorical variables were compared with Chisquare tests. Modified Poisson regression analysis was conducted to identify preoperative characteristics associated with the high-risk group [17]. This analysis estimates relative risk and $95 \%$ confidence intervals with use of robust error variances. Preoperative variables considered for model inclusion were those previously reported to be associated with the development of CPSP, including treatment group, surgery type, sex, age, race/ethnicity, marital status, income, baseline pain, receipt of opioid prescription in the 6 months prior to surgery, BDIII score, QuickDASH score, SF-36 physical component score, SF-36 mental component score, and positive SPTSS score. Model building sensitivity analyses included forward, backward, stepwise, and lasso selection algorithms. Model efficiency was determined with goodness-of-fit indices. Collinearity diagnostics for all variables considered for model inclusion were conducted.

\section{RESULTS}

\section{Sample Characteristics}

Of 131 randomized participants, 114 (83 CTR, 31 TFR) received treatment, provided at least 1 day of follow-up data after their operation, and were included in this secondary analysis. These 114 patients were the same cohort analyzed in the original clinical trial. Table 1 reports the preoperative characteristics for participants in high vs. low-risk categories for persistent post-surgical pain based on their average pain score on POD 10. Patients reporting an average pain score on POD $10<3$ were considered a part of the low-risk group while those reporting and average pain score on POD $10 \geq 3$ were considered part of the high-risk group. A total of 87 patients $(76.3 \%)$ were partitioned into the low-risk category, and 27 patients $(23.7 \%)$ were partitioned into the high-risk category. Overall, median (interquartile range [IQR]) pain duration in the high vs. low-risk category was 70 (25-160) vs. 14 (7-24) days.

\section{Cox Proportional Hazards Regression and Sensitivity Analyses}

We examined 15 acute pain descriptors including the pain clusters of the average, current, and worst pain trajectories (Fig. 1); mean and median of the average, current, and worst pain scores during the first ten postoperative days; average, current, and worst pain scores reported on POD 10; and linear slopes of the average, current, and worst pain trajectories using Cox proportional hazards regression controlling for operation type. Table 2 lists the corresponding hazard ratios (HRs) for each acute pain descriptor as a predictor of time to pain resolution. All of the acute pain descriptors except the median of the worst pain scores, and the linear slopes of the average, current, and worst pain trajectories were significantly associated with time to pain cessation. Of all the descriptors, average pain over the last $24 \mathrm{~h}$ reported on POD 10 was the best predictor of subsequent remote time to pain cessation. Every one-point increase in the average pain score on POD 10 was associated 
Table 1 Preoperative characteristics of patients according to average pain on postoperative day 10

\begin{tabular}{|c|c|c|c|}
\hline \multirow[t]{2}{*}{ Characteristic } & \multicolumn{2}{|l|}{ No. $(\%)^{\mathbf{a}}$} & \multirow[t]{2}{*}{$p$ value } \\
\hline & $\begin{array}{l}\text { Average pain on } \\
\text { postoperative day } 10<3\end{array}$ & $\begin{array}{l}\text { Average pain on } \\
\text { postoperative day } 10 \geq 3\end{array}$ & \\
\hline Patients, no. & $87(76.3 \%)$ & $27(23.7 \%)$ & \\
\hline Age, mean (SD), years & $60.8(10.6)$ & $55.7(11.0)$ & 0.03 \\
\hline Male gender & $71(84.5 \%)$ & $19(70.4 \%)$ & 0.10 \\
\hline Martial status & & & 0.48 \\
\hline Married & $40(47.6 \%)$ & $16(59.3 \%)$ & \\
\hline Never married & $3(3.6 \%)$ & $2(7.4 \%)$ & \\
\hline Living with someone but not married & $5(6.0 \%)$ & $2(7.4 \%)$ & \\
\hline Divorced or separated & $31(36.9 \%)$ & $7(25.9 \%)$ & \\
\hline Widowed & $5(6.0 \%)$ & $0(0.0 \%)$ & \\
\hline Race & & & 0.48 \\
\hline Caucasian & $62(73.8 \%)$ & $16(59.3 \%)$ & \\
\hline African American & $5(6.0 \%)$ & $3(11.1 \%)$ & \\
\hline Asian & $2(2.4 \%)$ & $0(0.0 \%)$ & \\
\hline Pacific Islander & $1(1.2 \%)$ & $1(3.7 \%)$ & \\
\hline American Indian & $4(4.8 \%)$ & $1(3.7 \%)$ & \\
\hline Other & $7(8.3 \%)$ & $4(14.8 \%)$ & \\
\hline \multicolumn{4}{|l|}{ Ethnicity } \\
\hline Hispanic or Latino & $13(15.5 \%)$ & $9(33.3 \%)$ & 0.04 \\
\hline Income (\%) & & & 0.17 \\
\hline$<\$ 10,000$ & $13(15.5 \%)$ & $2(7.4 \%)$ & \\
\hline$\$ 10,000-\$ 29,999$ & $31(36.9 \%)$ & $9(33.3 \%)$ & \\
\hline$\$ 30,000-\$ 49,999$ & $15(17.9 \%)$ & $5(18.5 \%)$ & \\
\hline$\$ 50,000-\$ 69,999$ & $10(11.9 \%)$ & $1(3.7 \%)$ & \\
\hline$\$ 70,000-\$ 89,000$ & $2(2.4 \%)$ & $1(3.7 \%)$ & \\
\hline$\$ 90,000+$ & $9(10.7 \%)$ & $3(11.1 \%)$ & \\
\hline Treatment group & & & 0.91 \\
\hline Placebo & $43(49.4 \%)$ & $13(48.2 \%)$ & \\
\hline Minocycline & $44(50.6 \%)$ & $14(51.9 \%)$ & \\
\hline Surgery type & & & 0.52 \\
\hline CTR & $60(71.4 \%)$ & $21(77.8 \%)$ & \\
\hline
\end{tabular}


Table 1 continued

\begin{tabular}{|c|c|c|c|}
\hline \multirow[t]{2}{*}{ Characteristic } & \multicolumn{2}{|l|}{ No. $(\%)^{a}$} & \multirow[t]{2}{*}{$p$ value } \\
\hline & $\begin{array}{l}\text { Average pain on } \\
\text { postoperative day } 10<3\end{array}$ & $\begin{array}{l}\text { Average pain on } \\
\text { postoperative day } 10 \geq 3\end{array}$ & \\
\hline TFR & $24(28.6 \%)$ & $6(22.2 \%)$ & \\
\hline Baseline pain, mean $(S D)^{b}$ & $3.2(2.4)$ & $6.1(2.6)$ & $<0.0001$ \\
\hline $\begin{array}{l}\text { Receipt of opioid prescription in the } \\
6 \text { months prior to surgery }\end{array}$ & $31(36.9 \%)$ & $19(70.4 \%)$ & $<0.01$ \\
\hline $\begin{array}{l}\text { Beck Depression Inventory-II score, mean } \\
(\mathrm{SD})^{\mathrm{c}}\end{array}$ & $8.9(9.0)$ & $18.4(11.9)$ & $<0.0001$ \\
\hline QuickDASH, mean $(S D)^{\mathrm{d}}$ & $36.3(21.7)$ & $55.7(17.1)$ & $<0.0001$ \\
\hline SF-36 Physical health, mean $(S D)^{e}$ & $58.8(7.2)$ & $52.9(7.6)$ & $<0.001$ \\
\hline SF-36 Mental health, mean $(S D)^{e}$ & $47.9(4.9)$ & $42.9(5.0)$ & $<0.0001$ \\
\hline Positive SPTSS Score for PTSD ${ }^{\mathrm{f}}$ & $24(27.6)$ & $20(74.1)$ & $<0.0001$ \\
\hline $\begin{array}{l}\text { Postoperative pain duration, median (IQR), } \\
\text { days }\end{array}$ & $14(7-24)$ & $70(25-160)$ & $<0.01$ \\
\hline
\end{tabular}

CTR carpal tunnel release, TFR trigger finger release, SPTSS screen for posttraumatic stress symptoms, PTSD posttraumatic stress disorder

${ }^{a}$ Percentages do not include patients with missing data

b Scale range, 1-10 (1, no pain; 10, pain as bad as you can imagine)

c Scale range, 0-63 (higher scores represent higher depressive symptoms)

d QuickDASH score range, 0-100; higher score indicates greater disability

e Short Form 36-item (SF-36) range, 0-100; 50 is average health

${ }^{f}$ SPTSS Score $>15$ cutoff for PTSD

with a $36.0 \%$ reduced rate of pain cessation. (HR, 0.64, 95\% CI 0.55-0.74, $p<0.001$ ).

There was a significant interaction between the average POD 10 pain score and treatment group ( $p$ value for interaction $=0.02$ ), and patients receiving minocycline were more likely to experience delayed pain resolution as POD 10 pain score increased compared to the group receiving placebo. No significant interactions were found between average POD 10 pain score and operation or SPTSS score (Table 3). After controlling for the interaction between treatment group and SPTSS, the average POD 10 pain score remained significantly associated with time to pain cessation (HR 0.64, 95\% CI 0.54-0.76, $P<0.0001)$.

\section{Determining Optimal Cutoff Value for the Best Acute Pain Descriptor}

We conducted a ROC curve analysis of persistent pain 90 days after surgery. Average pain reported on postoperative day (POD) \#10 was significantly associated with persistent pain 90 days after surgery (OR 1.74, 95\% CI $1.30-2.33, p$ value $<0.001)$. Overall, average pain over the last $24 \mathrm{~h}$ reported on POD \#10 was predictive of persistent pain 90 days after surgery $\quad(\mathrm{AUC}=0.85, \quad 95 \% \quad$ CI $0.77-0.93$, $p$ value $<0.0001)$. Based on the optimal Youden index $(J)$ value, $D$ value closest to the ideal point of the ROC plot, and equality point, patients reporting an average pain score on POD \#10 $\geq 3$ will have a $75.0 \%$ chance of having persistent pain 90 days after surgery. Conversely, patients 


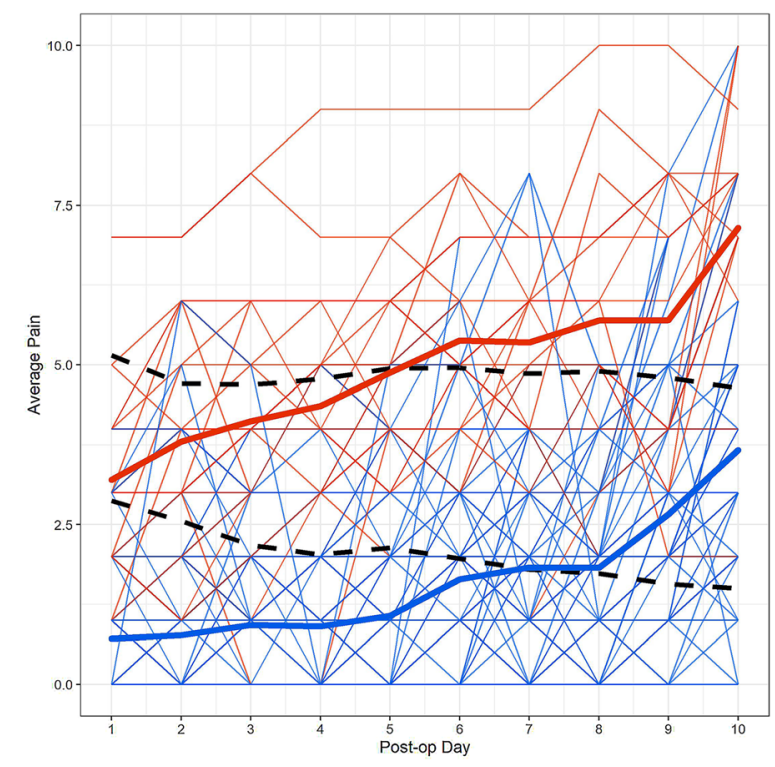

Fig. 1 Individual acute pain trajectories comparing the high vs. low acute pain clusters. Each line represents an individual patient's acute pain trajectory for the average pain scores reported over the first ten postoperative days. The solid bold lines represent the overall high vs. low pain cluster trajectory in red vs. blue, respectively. Previously calculated centroids used to classify patient trajectories are denoted by the black broken line

reporting an average pain score on POD \#10 $<3$ will have $84.6 \%$ chance of not developing persistent pain 90 days after surgery.

\section{Modified Poisson Regression Analysis}

Table 1 compares preoperative characteristics of low vs. high-risk patients based on average pain on POD 10 scores ( $\geq 3$ or less than 3 ). Twelve (46.2\%) high-risk patients vs. 4 (4.8\%) low-risk patients developed CPSP 90 days after their operation. Figure 2 shows the corresponding Kaplan-Meier curves stratified by risk category of average pain reported on POD 10 demonstrating good separation and maintenance of the proportional hazards assumption. Those in the high-risk group were slightly younger and more likely to report Hispanic or Latino ethnicity. These high-risk patients also reported significantly higher baseline pain severity, more often received opioid prescriptions in the 6 months prior to surgery, and exhibited greater psychological distress, disability, and reduced physical function.

We compared preoperative characteristics of participants between the high- and low-risk groups based on average pain reported on POD 10 ( $\geq 3$ or less than 3 ) in a modified Poisson regression analysis. There was a low chance of collinearity when examining variables considered for inclusion in the final model (tolerance range, 0.81-0.97; variance inflation factor range, 1.03-1.23). Several preoperative factors were associated with an increased risk of categorization to the high-risk acute pain cluster (Table 4). Elevated baseline pain severity (adjusted relative risk [ARR], 1.25; 95\% CI $1.07-1.48 ; p$ value $<0.01$ ) and Hispanic or Latino ethnicity (ARR 1.80; 95\% CI 1.05-3.07; $p$ value $=0.03$ ) were associated with categorization to the high-risk acute pain group. Higher SF-36 physical component scores, indicating better physical function, were associated with a decreased risk of categorization to the high-risk acute pain group (ARR, 0.94; 95\% CI 0.90-0.98; $p$ value $<0.01)$

\section{DISCUSSION}

We used a previously trained machine-learning classifier [10] and examined the first ten postoperative days of pain scores to sort patients undergoing CTR and TFR under local anesthesia into acute pain clusters [10, 18]. After comparison of these clusters and 12 additional acute pain descriptors, average pain intensity over the preceding 24-h reported on POD 10 was most predictive of remote time to pain resolution. Previously, the worst pain intensity over the past 24-h reported on POD 10 was reported as a significant immediate postoperative predictor of remote pain resolution, opioid cessation, and complete surgical recovery comparing the same series of acute pain descriptors [10]. This prior analysis was conducted among a mixed surgical cohort receiving general anesthesia, while we examined acute pain descriptors among patients undergoing hand surgery under local anesthesia. In our analysis, worst pain on POD 10 was the second-best acute pain descriptor of time to pain resolution. The difference in 
Table 2 Univariate sensitivity analyses of acute pain descriptors as determinants of remote pain cessation after hand surgery ${ }^{a}$

\begin{tabular}{|c|c|c|c|}
\hline Predictor & HR (95\% CI for HR) & AIC & $p$ value \\
\hline \multicolumn{4}{|c|}{ High pain cluster of pain trajectories } \\
\hline Average & $0.45(0.28-0.74)$ & 816.07 & 0.001 \\
\hline Current & $0.42(0.26-0.68)$ & 813.15 & $<0.001$ \\
\hline Worst & $0.32(0.22-0.48)$ & 797.15 & $<0.001$ \\
\hline \multicolumn{4}{|c|}{ Postoperative day 10 pain score } \\
\hline Average & $0.64(0.55-0.74)$ & 754.37 & $<0.001$ \\
\hline Current & $0.78(0.69-0.87)$ & 786.80 & $<0.001$ \\
\hline Worst & $0.76(0.68-0.84)$ & 763.82 & $<0.001$ \\
\hline \multicolumn{4}{|c|}{ Average pain scores over 10 days } \\
\hline Average & $0.53(0.41-0.67)$ & 795.00 & $<0.001$ \\
\hline Median & $0.46(0.36-0.59)$ & 780.28 & $<0.001$ \\
\hline \multicolumn{4}{|c|}{ Current pain scores over 10 days } \\
\hline Average & $0.57(0.45-0.72)$ & 800.61 & $<0.001$ \\
\hline Median & $0.60(0.48-0.74)$ & 800.14 & $<0.001$ \\
\hline \multicolumn{4}{|c|}{ Worst pain scores over 10 days } \\
\hline Average & $0.63(0.53-0.74)$ & 824.13 & $<0.001$ \\
\hline Median & $0.82(0.63-1.06)$ & 825.43 & 0.13 \\
\hline \multicolumn{4}{|c|}{ Linear slope of the pain trajectories } \\
\hline Average & $0.73(0.33-1.62)$ & 799.11 & 0.44 \\
\hline Median & $0.78(0.37-1.61)$ & 799.25 & 0.50 \\
\hline Worst & $0.60(0.33-1.08)$ & 796.91 & 0.09 \\
\hline
\end{tabular}

$H R$ hazard ratio, $C I$ confidence interval, AIC Akaike Information Criterion

${ }^{a}$ All results presented adjusted for type of operation

findings may reflect the uniformity of the operations performed in the current clinical trial. Similar to prior research, we found that pain intensity fluctuates widely in any given patient over the first ten postoperative days. Further, linear characterization of acute pain was a poor predictor of time to pain cessation consistent with prior research findings [10]. Overall, clinical assessment of pain intensity on POD10 was a better predictor of remote pain resolution than a series of other acute pain descriptors, thus validating and extending our prior work [10].

Acute pain severity is associated with an increased risk of persistent pain prevalence at varied time points after surgery. Among 72 elderly patients who underwent surgery for hip fractures, pain with movement assessed 2 and 4 weeks after surgery was significantly associated with persistent pain at 8 weeks after surgery [19]. Similarly, severe acute postoperative pain after Cesarean section has been associated with the development of CPSP [20]. Acute pain 
Table 3 Subgroup analyses for remote pain cessation after hand surgery

\begin{tabular}{lcc}
\hline Subgroup & $\begin{array}{l}\text { HR (95\% CI } \\
\text { for HR) }\end{array}$ & $\begin{array}{c}\boldsymbol{p} \text { value for } \\
\text { interaction }\end{array}$ \\
\hline $\begin{array}{l}\text { Effect of one-unit change in } \\
\text { average postoperative day }\end{array}$ & 0.02 \\
10 pain score by & & \\
treatment group & & \\
Placebo & 0.74 & \\
& $(0.62-0.90)$ & \\
Minocycline & 0.52 & \\
& $(0.41-0.66)$ & \\
Effect of one-unit change in & \\
average postoperative day & \\
10 pain score by & \\
operation & \\
CTR & 0.68 \\
& $(0.58-0.80)$ \\
TFR & 0.51 \\
& $(0.37-0.70)$
\end{tabular}

Effect of 1-unit change in average postoperative day 10 pain score by SPTSS score

$$
\begin{array}{lr}
\text { SPTS }<16 \text { (no PTSD } & 0.59 \\
\text { subgroup) } & (0.45 \\
\text { SPTSS } \geq 16 \text { (PTSD } & 0.65 \\
\text { subgroup) } & (0.53
\end{array}
$$

Average post-operative day

10 pain score ${ }^{\mathrm{a}}$

$$
\begin{array}{ll}
0.64 & <0.0001 \\
(0.54-0.76) &
\end{array}
$$

$H R$ hazard ratio, $C I$ confidence interval, CTR carpal tunnel release, TFR trigger finger release, SPTSS screen for posttraumatic stress symptoms, PTSD post-traumatic stress disorder

${ }^{\text {a }}$ Adjusted for interaction of treatment group and SPTSS score

intensity assessed on POD 3 after cardiac surgery was associated with both the presence and intensity of postoperative non-anginal pain 24 months after surgery [21]. Among 123 patients undergoing breast cancer surgery, group-based trajectory modeling of pain

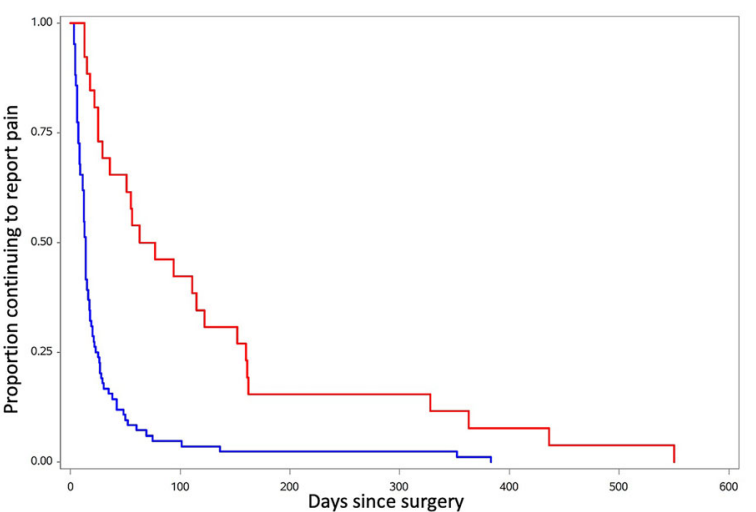

Fig. 2 High vs. low average pain reported on postoperative day 10 and post-operative pain resolution. Time to pain resolution in days comparing patients reporting an average pain score on postoperative day 10 of $\geq 3$ (red line) vs. those reporting a score $<3$ (blue line). Log rank $p$ value $<0.0001$

Table 4 Preoperative risk factors for high average pain reported on post-operative day $10^{a}$

\begin{tabular}{lllr}
\hline Characteristic & ARR & $\begin{array}{l}\text { 95\% confidence } \\
\text { interval }\end{array}$ & $\boldsymbol{p}$ value \\
\hline $\begin{array}{l}\text { SF-36 Physical } \\
\quad \text { Health score }\end{array}$ & 0.94 & $(0.90-0.98)$ & $<0.01$ \\
$\begin{array}{l}\text { Baseline pain } \\
\text { c }\end{array}$ & 1.25 & $(1.07-1.48)$ & $<0.01$ \\
$\begin{array}{l}\text { Hispanic or Latino } \\
\text { ethnicity }\end{array}$ & 1.80 & $(1.05-3.07)$ & 0.03 \\
$\begin{array}{l}\text { Positive SPTSS score } \\
\text { for PTSD }\end{array}$ & 2.30 & $(0.94-5.59)$ & 0.06 \\
\hline
\end{tabular}

$A R R$ adjusted relative risk, SPTSS screen for post-traumatic stress symptoms, PTSD post-traumatic stress disorder

${ }^{a}$ Modified Poisson regression

b Every 1-point increase in the SF-36 Physical Health score

${ }^{c}$ Every 1-point increase in the Numeric Rating Scale of Pain

intensity during movement over the first 7 postoperative days yielded mild, moderate, and severe pain clusters. The severe pain cluster was at significantly increased risk of elevated pain intensity assessed 6 months post-op. Our findings add to this research examining a group of patients undergoing hand surgery under local 
anesthesia. We conducted a thorough comparison of a series of acute pain descriptors to identify average pain on POD 10 as the best predictor of CPSP compared to other more sophisticated acute pain metrics. We further report a significant association between this descriptor and persistent post-surgical pain rather than the association with postoperative pain prevalence at any given time point. Assessing postoperative pain resolution in our study may be less prone to patient-reporting bias towards higher pain severity scores in both the acute and chronic phases, which is likely to overestimate the significance of associations. For added clinical significance, we report the optimal cutoff value for average pain on POD 10 with the capacity to discriminate between patients at low or high risk of prolonged postoperative pain.

These findings show the value of pain assessment long after hospital discharge in patients undergoing either minor or major operations is highlighted by these findings. Measurement of pain intensity on POD 10 can be readily implemented in current clinical practice. POD 10 is around the time patients return for a postoperative assessment. Thus, a deliberate pain assessment would facilitate early identification of patients requiring more intensive postoperative pain management or other multimodal pain interventions. We found improved discriminative capacity for average pain on POD 10 to predict persistent pain 90 days after CTR or TFR compared to prior research [10]. Future work to replicate our current findings and assess additional outcomes including postoperative opioid cessation and surgical recovery are needed to understand the importance of this acute pain descriptor. In addition, future work should begin to explore candidate early interventions to improve 90-day outcomes among that group of patients identified as high risk by POD 10 average pain intensity. In our previous studies of perioperative minocycline and gabapentin, we observed that many patients were reluctant to participate in trials of preoperative or perioperative interventions to reduce the chance of developing chronic pain for two commonly cited reasons: (1) they believed themselves unlikely to be affected by such an outcome; and (2) they already have many very practical issues competing for their attention and time in the days leading up to their surgery. In contrast, patients experiencing greater-than-expected postoperative pain severity, especially by POD 10 , are often quite eager to explore interventions that may ultimately prove in the future to reduce future pain and suffering. Efforts to replicate the significance of acute pain intensity on POD 10 in predicting postoperative pain resolution across a variety of operations is likely to improve identification of high-risk patients before pain becomes chronic and refractory to intervention.

Another application is to consider subacute pain intensity on POD 10 as a surrogate endpoint for the development of CPSP. Thus, average pain intensity reported on POD 10 may be considered a surrogate endpoint for the development of CPSP. Preliminary or pilot clinical trials could be conducted while sparing resources. Our study involved 3917 assessments of postoperative pain with longitudinal followup to 2 years after the operation. A surrogate endpoint would allow for a significant decrease in resources for follow-up assessments. However, an important question is whether interventions that significantly improve pain intensity on POD 10 consistently decrease the incidence of CPSP.

Subgroup analysis revealed a significant interaction of the best acute pain descriptor with treatment group, and patients receiving minocycline reporting higher average pain on POD10 were significantly more likely to experience remote delayed postoperative pain resolution. These findings may reflect two distinct phenomena. First, patients reporting higher average pain on POD 10 may be globally refractory to positive outcomes to perioperative pain interventions given patient-specific preoperative characteristics. Patients in our study reporting higher levels of preoperative pain severity were more likely to report higher acute postoperative pain. Preoperative pain has been reported as a risk factor for both increased pain and CPSP [22]. It is possible that pre-existing pain stimuli predisposes a patient to pain sensitization resulting from surgical tissue trauma 
[22]. However, when examining patients undergoing total knee arthroplasty, a systematic review graded the association between preoperative pain and CPSP as low quality and reported an inverse relationship between higher levels of preoperative pain associated with lower pain levels after total knee arthroplasty [23]. Also, patients in our study reporting lower levels of preoperative physical function were more likely to be categorized in the high-risk acute pain group. In a retrospective analysis of 91 patients undergoing single-level, primary anterior cervical discectomy and fusion, patients with reduced preoperative physical function reported increased acute pain during their hospital stay. However, long-term improvements in neck and arm pain from were comparable from 6 weeks to 1 year after surgery [24]. Patients undergoing primary CTR report significant rapid and sustained improvement in physical function, and a similar relationship with postoperative pain is possible whereby preoperative physical function is not significantly associated with CPSP [25].

Taken together, the interaction between the high-risk acute pain group and treatment assignment is unlikely solely related to preoperative patient-specific risk factors. Unplanned re-admissions and emergency department visits after hand surgery commonly relate to uncontrolled postoperative pain, and operative risks factors for unplanned readmissions include increasing operative time, and procedures performed by surgeons other than plastic or orthopedic surgeons [26, 27]. Patients categorized to high acute pain based on the average POD 10 score may represent a confluence of risk factors both intrinsic and extrinsic to the patients, and related to pre-, intra-, and postoperative factors. This score may represent the confluence of a patient's response to surgery tissue trauma taking both intrinsic and extrinsic factors into consideration. Therefore, the significantly reduced response to minocycline in the high-risk acute pain group may indicate an altered subacute trajectory that ultimately predisposes to delayed remote postoperative pain resolution.

Previously, a significant association between preoperative depressive symptoms and postoperative scar pain after CTR was reported. Ultimately, a small percentage of variance was explained with this psychological factor further pointing to the importance of other mechanisms in the development of CPSP [28]. In our study, psychological factors were not significantly associated with categorization to the high-risk acute group. Rather, Hispanic or Latino ethnicity was a significant predictor of higher reported average pain intensity on POD 10. Prior research reported that Hispanics were less likely to receive analgesia for acute pain in US emergency departments [29]. Similarly, Hispanics receive less opioids during hospitalization for acute pancreatitis compared to nonHispanic white patients [30]. Given that our study represents a randomized controlled trial of an analgesic intervention, the disparity may not be explained entirely by a disparity in analgesic prescribing. Future research is needed to determine whether this disparity is consistently seen across different operations.

Our study possesses a number of strengths. The frequency of daily postoperative assessments provides granularity in the assessment of postoperative pain resolution. With extended longitudinal follow-up up to 2 years after CTR or TFR, we did not rely on reports of pain intensity at discreet time points such as 3,6 , or 12 months after the operation. Our definition of pain resolution required a sustained remission of pain. Also, we aimed to test a specific hypothesis first suggested by prior work in a separate and distinct surgical cohort, that acute pain assessment on POD10 was a better predictor of future pain outcomes than all other competing metrics. Our study identified an acute pain descriptor that is easy to assess in real-time and the ease of clinical implementation has the potential to substantially improve perioperative pain management. More complex acute pain descriptors requiring data collection and processing may decrease ease of implementation and reduce test interpretability.

Our study has limitations as a secondary analysis of a clinical trial. The trial did not specify a standard postoperative pain management protocol beyond dosing of minocycline. Although operative details were standardized, it is unclear whether the rate of non-opioid and 
opioid analgesic use was consistent across treatment groups after the operation, which could ultimately affect postoperative pain resolution. Further, the analysis was limited by a modest sample size, and future work to replicate findings in larger surgical cohorts is needed. However, $14 \%$ of patients in our study reported persistent pain 90 days after surgery, which represents a sizeable group. The patients in our study also represent a predominately male veteran cohort. Higher rates of mental health disorders and social stress occur among veterans. Further, carpal tunnel or trigger finger typically affects women more commonly than men. Prior work noted that female sex was a risk factor for categorization to a high acute pain cluster in a mixed surgical cohort [10], and future research should include non-veteran cohorts with a higher percentage of females.

\section{CONCLUSIONS}

Our study findings suggest average pain intensity over the past $24 \mathrm{~h}$ reported on POD 10 is a significant postoperative predictor of delayed pain resolution after CTR or TFR. This measure can be quickly assessed in person, via phone, or through digital app-based assessments to identify patients at high risk for prolonged postoperative pain. These high-risk patients would likely benefit from early pain intervention, closer follow-up, and initiation of multimodal pain treatments. Continued research in larger surgical cohorts comparing a series of acute pain descriptors is needed to validate the importance of postoperative pain intensity reported on POD 10.

\section{ACKNOWLEDGEMENTS}

The authors would like to thank the participants of the study for their contribution to the research.

Funding. R. Hah reported grant funding from the National Institutes of Health $(\mathrm{NIH})$ National Institute on Drug Abuse (R01DA045027) to support the study. Dr. Curtin received support through a RR\&D pilot grant RX000487 from the U.S. Department of Veterans Affairs Rehabilitation and Research and Development Service to support the study. The journal's Rapid Service Fee was funded by the Department of Anesthesiology, Perioperative, and Pain Medicine at Stanford University. The funders had no role in the design and conduct of the study; collection, management, analysis, and interpretation of the data; preparation, review, or approval of the manuscript; and decision to submit the manuscript for publication. The contents of this work do not represent the views of the U.S. Department of Veterans Affairs or the United States government.

Authorship. All named authors meet the International Committee of Medical Journal Editors (ICJME) criteria for authorship for this article, take responsibility for the integrity of the work as a whole, and have given their approval for this version to be published.

Authors' Contributions. Dr. Hah and Dr. Curtin had full access to all of the data in the study and take responsibility for the integrity of the data and the accuracy of the data analysis. Concept and design: Hah, Curtin, Carroll. Acquisition, analysis, or interpretation of data: Hah, Nwaneshiudu, Cramer, Curtin, Carroll. Drafting of the manuscript: Hah. Critical revision of the manuscript for important intellectual content: Hah, Nwaneshiudu, Cramer, Curtin, Carroll. Statistical analysis: Hah, Cramer. Obtained funding: Hah, Curtin. Administrative, technical, or material support: Hah, Curtin. Supervision: Hah, Curtin.

Disclosures. Jennifer M. Hah, Chinwe A. Nwaneshiudu, Eric M. Cramer, Ian R. Carroll and Catherine M. Curtin do not have any disclosures to report.

Compliance with Ethics Guidelines. The study was approved by the Stanford University IRB \#21,794 (clinicaltrials.gov identifier NCT02051296, RX000487) and all patients provided informed consent to participate. A detailed description of the study protocol, demographics, measures, and methods has been 
published [11]. The study was performed in accordance with the Helsinki Declaration of 1964 , and its later amendments.

Data Availability. The data are available upon reasonable request at the discretion of the authors. The data are not publicly available due to privacy restrictions of research participants.

Open Access. This article is licensed under a Creative Commons Attribution-NonCommercial 4.0 International License, which permits any non-commercial use, sharing, adaptation, distribution and reproduction in any medium or format, as long as you give appropriate credit to the original author(s) and the source, provide a link to the Creative Commons licence, and indicate if changes were made. The images or other third party material in this article are included in the article's Creative Commons licence, unless indicated otherwise in a credit line to the material. If material is not included in the article's Creative Commons licence and your intended use is not permitted by statutory regulation or exceeds the permitted use, you will need to obtain permission directly from the copyright holder. To view a copy of this licence, visit http://creativecommons.org/licenses/bync/4.0/.

\section{REFERENCES}

1. Glare P, Aubrey KR, Myles PS. Transition from acute to chronic pain after surgery. Lancet. 2019;393(10180):1537-46.

2. Belze O, Remerand F, Laulan J, et al. Chronic pain after carpal tunnel surgery: epidemiology and associated factors. Ann Fr Anesth Reanim. 2012;31(12):e269-74.

3. Boya H, Ozcan O, Ozteki NH. Long-term complications of open carpal tunnel release. Muscle Nerve. 2008;38(5):1443-6.

4. da Costa VV, de Oliveira SB, Fernandes Mdo C, Saraiva RA. Incidence of regional pain syndrome after carpal tunnel release. Is there a correlation with the anesthetic technique? Rev Bras Anestesiol. 2011;61(4):425-33.
5. Johnson SP, Chung KC, Zhong L, et al. Risk of prolonged opioid use among opioid-naive patients following common hand surgery procedures. J Hand Surg Am. 2016;41(10):947-57e3.

6. Kehlet H, Jensen TS, Woolf CJ. Persistent postsurgical pain: risk factors and prevention. Lancet. 2006;367(9522):1618-25.

7. Chapman CR, Donaldson GW, Davis JJ, Bradshaw DH. Improving individual measurement of postoperative pain: the pain trajectory. J Pain. 2011;12(2):257-62.

8. Lavand'homme $\mathrm{P}$. The progression from acute to chronic pain. Curr Opin Anaesthesiol. 2011;24(5): 545-50.

9. Fajardo M, Kim SH, Szabo RM. Incidence of carpal tunnel release: trends and implications within the United States ambulatory care setting. J Hand Surg Am. 2012;37(8):1599-605.

10. Hah JM, Cramer E, Hilmoe H, et al. Factors associated with acute pain estimation, postoperative pain resolution, opioid cessation, and recovery: secondary analysis of a randomized clinical trial. JAMA Netw Open. 2019;2(3):e190168.

11. Curtin CM, Kenney D, Suarez P, et al. A doubleblind placebo randomized controlled trial of minocycline to reduce pain after carpal tunnel and trigger finger release. J Hand Surg Am. 2017;42(3): $166-74$.

12. Cleeland CS, Ryan KM. Pain assessment: global use of the Brief Pain Inventory. Ann Acad Med Singap. 1994;23(2):129-38.

13. Caspi Y, Carlson EB, Klein E. Validation of a screening instrument for posttraumatic stress disorder in a community sample of Bedouin men serving in the Israeli Defense Forces. J Trauma Stress. 2007;20(4):517-27.

14. Beck AT, Steer RA. Internal consistencies of the original and revised Beck Depression Inventory. J Clin Psychol. 1984;40(6):1365-7.

15. Polson K, Reid D, McNair PJ, Larmer P. Responsiveness, minimal importance difference and minimal detectable change scores of the shortened disability arm shoulder hand (QuickDASH) questionnaire. Man Ther. 2010;15(4):404-7.

16. Laucis NC, Hays RD, Bhattacharyya T. Scoring the SF-36 in orthopaedics: a brief guide. J Bone Joint Surg Am. 2015;97(19):1628-34.

17. Zou G. A modified Poisson regression approach to prospective studies with binary data. Am J Epidemiol. 2004;159(7):702-6. 
18. Parthipan A, Banerjee I, Humphreys K, et al. Predicting inadequate postoperative pain management in depressed patients: a machine learning approach. PLoS ONE. 2019;14(2):e0210575.

19. Goto K, Kataoka H, Honda A, et al. Factors affecting persistent postoperative pain in patients with hip fractures. Pain Res Manag. 2020;2020:8814290.

20. Yimer $\mathrm{H}$, Woldie $\mathrm{H}$. Incidence and associated factors of chronic pain after caesarean section: a systematic review. J Obstet Gynaecol Can. 2019;41(6): 840-54.

21. Choiniere M, Watt-Watson J, Victor JC, et al. Prevalence of and risk factors for persistent postoperative nonanginal pain after cardiac surgery: a 2-year prospective multicentre study. CMAJ. 2014;186(7):E213-23.

22. Fregoso G, Wang A, Tseng K, Wang J. Transition from acute to chronic pain: evaluating risk for chronic postsurgical pain. Pain Phys. 2019;22(5): 479-88.

23. Harmelink KEM, Zeegers A, Hullegie W, Hoogeboom TJ, van der-Nijhuis Sanden MWG, Staal JB. Are there prognostic factors for one-year outcome after total knee arthroplasty? A Systematic Review. J Arthroplasty. 2017;32(12):3840-53.

24. Parrish JM, Jenkins NW, Narain AS, Hrynewycz NM, Brundage TS, Singh K. Postoperative pain, narcotics consumption, and patient-reported outcomes based on PROMIS physical function following a singlelevel anterior cervical discectomy and fusion. Spine (Phila Pa 1976). 2020;45(17):E1091-6.

25. Niedermeier SR, Pettit RJ, Frantz TL, Colvell KHMA. Early postoperative improvement in sleep and pain after carpal tunnel release. Hand (N Y). 2020;15(3): 311-4.

26. Donato DP, Kwok AC, Bishop MO, Presson AP, Agarwal JP. Unplanned readmission in outpatient hand surgery: an analysis of 23,613 patients in the NSQIP Data Set. Eplasty. 2017;17:e36.

27. Menendez ME, Ring D. Emergency department visits after hand surgery are common and usually related to pain or wound issues. Clin Orthop Relat Res. 2016;474(2):551-6.

28. Kim JK, Kim YK. Predictors of scar pain after open carpal tunnel release. J Hand Surg Am. 2011;36(6): 1042-6.

29. Lee P, Le Saux M, Siegel R, et al. Racial and ethnic disparities in the management of acute pain in US emergency departments: Meta-analysis and systematic review. Am J Emerg Med. 2019;37(9): 1770-7.

30. Wu BU, Butler RK, Chen W. Factors associated with opioid use in patients hospitalized for acute pancreatitis. JAMA Netw Open. 2019;2(4):e191827. 